\title{
Características clínicas, diagnósticas y pronósticas de pacientes con neumonía por Pneumocystis jiroveci en individuos infectados por virus de inmunodeficiencia humana e individuos inmunocomprometidos por otra etiología
}

\author{
Inés Cerón, Ricardo Rabagliati, Javiera Langhaus, Felipe Silva, Ana M. Guzmán y Marcela Lagos
}

Pneumocystis jiroveci pneumonia: comparative study of cases in HIV-infected patients and immunocompromised non-HIV-infected patients

Background: Although P. jiroveci pneumonia affects immunocompromised (IC) patients of any etiology, clinical features and prognostic outcomes are different depending if they are patients with HIV infection or other causes of IC. Objectives: To compare clinical and laboratory features as well as outcomes of $P$. jiroveci pneumonia in HIV versus non-HIV patients. Methods: Retrospective review of clinical records of HIV and non-HIV patients with P. jiroveci pneumonia managed at the Hospital Clínico Universidad Católica in Santiago, Chile, between 2005 and 2007. Results: We included $28 \mathrm{HIV}$ and 45 non-HIV patients with confirmed $P$. jiroveci pneumonia. The non-HIV population was older ( 65 vs 36,2 years, $p<0,01)$, had shorter duration of symptoms (7 [1-21] vs 14 [2-45] days, $\mathrm{p}<0,01)$, required more invasive techniques $(60 \mathrm{vs} 21 \%, \mathrm{p}<0,01)$ and RT-PCR to confirm the diagnosis (93 vs $68 \%, \mathrm{p}<0,01)$, were more frequently treated at intensive care units $(58$ vs. $25 \%, \mathrm{p}<0,01)$ requiring artificial ventilation ( 56 vs $11 \%, \mathrm{p}<0,01)$, and had a higher attributable mortality $(33 \%$ vs $0 \%, \mathrm{p}<0,01)$. Conclusions: Our study confirmed that $P$. jiroveci pneumonia in non-HIV IC patients is more severe, more difficult to diagnose and has higher mortality that in HIV patients. Therefore, it is mandatory to optimize diagnostic and therapeutic strategies for this patients group.

Key words: Pneumocystis jiroveci, pneumonia, pneumocystosis, HIV, immunosuppression.

Palabras clave: Pneumocystis jiroveci, neumonía, pneumocistosis, VIH, inmunosupresión.

\section{Introducción}

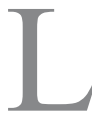

a neumonía por Pneumocystis jiroveci (pneumocistosis) es una infección oportunista potencialmente mortal que puede afectar a un amplio grupo de individuos inmunocomprometidos, incluyendo pacientes con infección por VIH con recuento de linfocitos T CD4+ $<200$ céls $/ \mathrm{mm}^{3}$; pero también está descrita en pacientes inmunocomprometidos por otras etiologías ${ }^{2}$ como trasplantes de órgano sólido ${ }^{3,4} \mathrm{y}$ de precursores hematopoyéticos $^{5,6}$, neoplasias ${ }^{7,8}$, receptores de quimioterapia y/o glucocorticoides ${ }^{9,10}$, siendo este último el primer grupo de pacientes en que se describió la infección ${ }^{11,12}$.

Según datos reportados en la literatura científica, la expresión clínica de la enfermedad y el pronóstico son diferentes en ambos grupos. En pacientes inmunocomprometidos no infectados por VIH, el diagnóstico es más dificultoso por la presencia de variables confundentes: infecciones concomitantes, uso de fármacos citotóxicos y compromiso pulmonar por enfermedad de base, describiéndose una mortalidad cercana a $40 \%$, cifra muy superior al $10 \%$ descrito en pacientes con infección por $\mathrm{VIH}^{13-15}$.

Pese al incremento en el número de pacientes inmunocomprometidos no infectados por VIH susceptibles de adquirir esta infección, existen pocos estudios que analicen comparativamente la neumonía por $P$. jiroveci en ambos grupos ${ }^{13,16-20}$. En el medio nacional, la información también es escasa, con poca representatividad de pacientes no infectados por $\mathrm{VIH}^{21,22}$.

El objetivo del presente estudio fue comparar las características clínicas, de laboratorio y pronóstico de las pneumocistosis en pacientes con infección por VIH versus inmunocomprometidos por otras etiologías.

\section{Pacientes y Método}

\section{Diseño}

Estudio retrospectivo, comparativo, de casos confirmados de neumonía por $P$. jiroveci en pacientes con infección por VIH vs inmunocomprometidos de otra
Pontificia Universidad Católica de Chile, Santiago. Escuela de Medicina Departamento de Enfermedades Infecciosas del Adulto (IC, RR,). Internos de la Escuela de Medicina (JL,FS).

Facultad de Medicina Departamento Laboratorios Clínicos (AMG, ML).

Lugar de realización del estudio: Hospital Clínico Pontificia Universidad Católica de Chile.

Los autores declaran no tener conflictos de interés. No se contó con financiamiento para la realización de este estudio.

Recibido: 8 de noviembre de 2013 Aceptado: 12 de mayo de 2014

Correspondencia a: Inés Cerón Araya. iceron76@yahoo.com 
etiología, ingresados en el Hospital Clínico de la Pontificia Universidad Católica de Chile entre los años 2005 y 2007.

\section{Institución}

Hospital universitario de nivel terciario de atención, ubicado en la ciudad de Santiago, Chile, donde anualmente ingresan alrededor de 25.000 pacientes y que cuenta con cerca de 500 camas. La unidad de onco-hematología es centro de referencia de pacientes procedentes de otras instituciones para tratamientos con quimioterapias y además, en ella se realizan trasplantes de precursores hematopoyéticos. El centro además cuenta con un programa de trasplantes de órganos sólidos que incluye riñón, hígado y corazón. Por su parte, durante los años del estudio el Departamento de Enfermedades Infecciosas del Adulto tenía alrededor de 350 pacientes con infección por VIH en control.

\section{Criterios de inclusión y exclusión}

Se incluyeron todos los pacientes $>15$ años con neumonía por $P$. jiroveci, definida como tal por sus médicos tratantes, dado la presencia de síntomas respiratorios bajos, imágenes pulmonares compatibles en radiografía o tomografía computada de tórax, y detección de $P$. jiroveci por técnica de inmunofluorescencia directa (IFD) y/o reacción de polimerasa en cadena, en tiempo real (RPC$\mathrm{TR}$ ) en una o más muestras respiratorias (expectoración inducida, gárgara, aspirado nasofaríngeo, aspirado endotraqueal o lavado bronquio-alveolar), según lo descrito en los reportes publicados ${ }^{23,24}$. Se excluyeron aquellos casos catalogados por sus tratantes como colonización o aquellos con registros clínicos incompletos que no permitieron dilucidar si se trataba de una colonización o de una infección clínica.

\section{Registro de datos}

Los episodios de neumonía por $P$. jiroveci fueron identificados, retrospectivamente, mediante la revisión de fichas clínicas. Para pre-seleccionar los pacientes a revisar, se solicitó al laboratorio de la institución el registro de todos los pacientes que hubieren presentado IFD y/o RPC-TR positivas para $P$. jiroveci en muestras respiratorias. Posteriormente, se revisaron las fichas clínicas de los pacientes pre-seleccionados para la identificación de los casos que cumplieran con los criterios de inclusión y exclusión antes mencionados. Una vez identificados, se procedió al registro en una base de datos pre-diseñada, consignando datos demográficos, factores predisponentes (enfermedad de base, tratamientos inmunosupresores, recuento de $\mathrm{LT} \mathrm{CD} 4+$, terapia anti-retroviral (TARV) y uso de profilaxis anti- $P$. jiroveci), características clínicas de cada episodio, co-infección con otros patógenos pulmonares, datos de laboratorio (general, microbiológico e imágenes), tratamientos utilizados y evolución clínica.

\section{Definiciones}

La definición de mortalidad atribuible se basó en lo registrado en las fichas clínicas por los propios tratantes. En aquellos casos en los que no estaba claramente especificada la causa del fallecimiento, se consideró como atribuible a la infección por $P$. jiroveci si la falla respiratoria fue un factor contribuyente en la progresión a la muerte y el diagnóstico y/o tratamiento de neumonía por $P$. jiroveci fue concomitante con el desarrollo de la insuficiencia respiratoria.

\section{Análisis estadístico}

Los datos fueron analizados utilizando los programas SPSS ${ }^{\circledR}$ y MedCalc ${ }^{\circledR}$. Se utilizó descriptores estadísticos habituales (promedio, desviación standard, mediana y rango). Se utilizó la prueba de t de Student y el test de $\chi^{2}$ en las comparaciones y para el análisis de sobrevida se usó curva de Kaplan Meier. Se consideró un valor $\mathrm{p}<0,05$ para la significancia estadística de las diferencias observadas.

\section{Resulltados}

Se preseleccionaron 94 pacientes adultos con IFD y/o RPC-TR de $P$. jiroveci positivas en muestras respiratorias. De éstos, se excluyeron 21 pacientes: cuatro por haber sido catalogados por sus tratantes como colonizaciones y 17 por registros clínicos incompletos o traslados a otros centros a completar tratamiento que no permitían definir si se había tratado de una infección o de una colonización o no se lograba conocer el desenlace final, por lo que finalmente, se incluyeron en el análisis 28 pacientes con infección por VIH y 45 no infectados por VIH, todos con diagnóstico de certeza de neumonía por $P$. jiroveci (Figura 1).

\section{Datos bio-demográficos}

El grupo de infectados por VIH se caracterizó por ser más joven, con una mediana de edad de 36,5 [26-79] vs $62[21-84]$ años $(\mathrm{p}<0,001)$ y tener predominio de sexo masculino respecto del grupo no infectado por $\mathrm{VIH}(85,7$ vs $51,1 \% ; \mathrm{p}=0,002)$.

\section{Co-morbilidades}

En el grupo de infectados por VIH la mediana de LTCD4 fue de 20 céls $/ \mathrm{mm}^{3}$ (rango: 2,6-180). En 17 pacientes $(60,7 \%)$ el episodio correspondió al debut de la enfermedad. Ocho de 11 (73\%) de los pacientes seropositivos conocidos estaba sin profilaxis anti- $P$. jiroveci. Trece pacientes $(40,6 \%)$ presentaban simultáneamente o tenían el antecedente de otras enfermedades marcadoras de SIDA: candidiasis esofágica (7 casos) y neumonía por $P$. jiroveci previa (3 casos). Sólo un paciente tenía 
patología pulmonar de base (tromboembolismo pulmonar y alveolitis fibrosante). Un paciente recibía adicionalmente tratamiento con prednisona; ningún otro paciente tenía concomitantemente alguna patología conocida que comprometiese su estado inmune. En el grupo sin infección por VIH, las patologías de base más prevalentes fueron: neoplasia hematológica $(28,9 \%)$, fibrosis pulmonar $(24,4 \%)$, enfermedad autoinmune (22,2\%) y tumor sólido (20\%), presentando algunos pacientes más de una enfermedad de base. Todos los pacientes contaban con test de ELISA para VIH negativo. Respecto al uso de inmunosupresores, 31 pacientes $(68,9 \%)$ recibían tratamiento corticosteroidal (28 prednisona y 3 metilprednisolona) y $9(20 \%)$ recibían quimioterapia. Entre aquellos que recibían corticosteroides había pacientes que adicionalmente utilizaban uno o más inmunosupresores: azatioprina ( 7 pacientes), ciclofosfamida (n: 2), rapamicina (n: 2), metotrexato (n: 3), infliximab (n: 1) o quimioterapia (n: 9). El 35\% de los pacientes (16/45) presentaba algún tipo de patología pulmonar de base: fibrosis pulmonar (n: 11), cáncer pulmonar (n: 3) y limitación crónica al flujo aéreo (LCFA) (n: 2). El 97,5\% de los pacientes (44/45) no recibía profilaxis anti- $P$. jiroveci.

\section{Expresión clínica de la pneumocistosis}

Respecto a la presentación clínica, los pacientes con infección por VIH presentaban con mayor frecuencia fiebre más elevada y tos seca mientras que en los pacientes no infectados por VIH predominaban la disnea y el examen pulmonar alterado (Tabla 1). La duración de los síntomas, previo a la consulta en un centro de salud, fue mayor en el grupo portador de VIH sobre el no infectado por VIH (14 [2-45] vs 7 [1-21] días $\mathrm{p}<0,001)$; pero por otro lado, la latencia entre la primera atención médica y la confirmación etiológica del diagnóstico fue mayor en el grupo no infectado por VIH (5 [1-25] vs 3 [1-10] días, $\mathrm{p}<0,001)$.

El grupo no portador de VIH presentó con mayor frecuencia infección pulmonar concomitante por otros agentes infecciosos $(42,2$ vs $17,9 \%, p=0,03)$, sin diferencias entre los distintos patógenos, salvo la mayor presencia de co-infección por micobacterias en el grupo de infectados por VIH (Tabla 2).

\section{Hallazgos de laboratorio}

Las características de laboratorio y radiológicas se resumen en la Tabla 3 donde destaca la mayor heterogeneidad en el patrón radiográfico del grupo no infectado por VIH y que el mismo grupo requirió más frecuentemente la realización de LBA y RPC-TR para llegar al diagnóstico. En 48 pacientes (14 en el grupo con infección por VIH (50\%) y 34 en el grupo no infectado por VIH $(75,6 \%)$ se realizó estudio etiológico tanto por técnica de IFD como por RPC-TR. En los pacientes del grupo infectado por VIH con estudio por ambas técnicas, el diagnóstico se

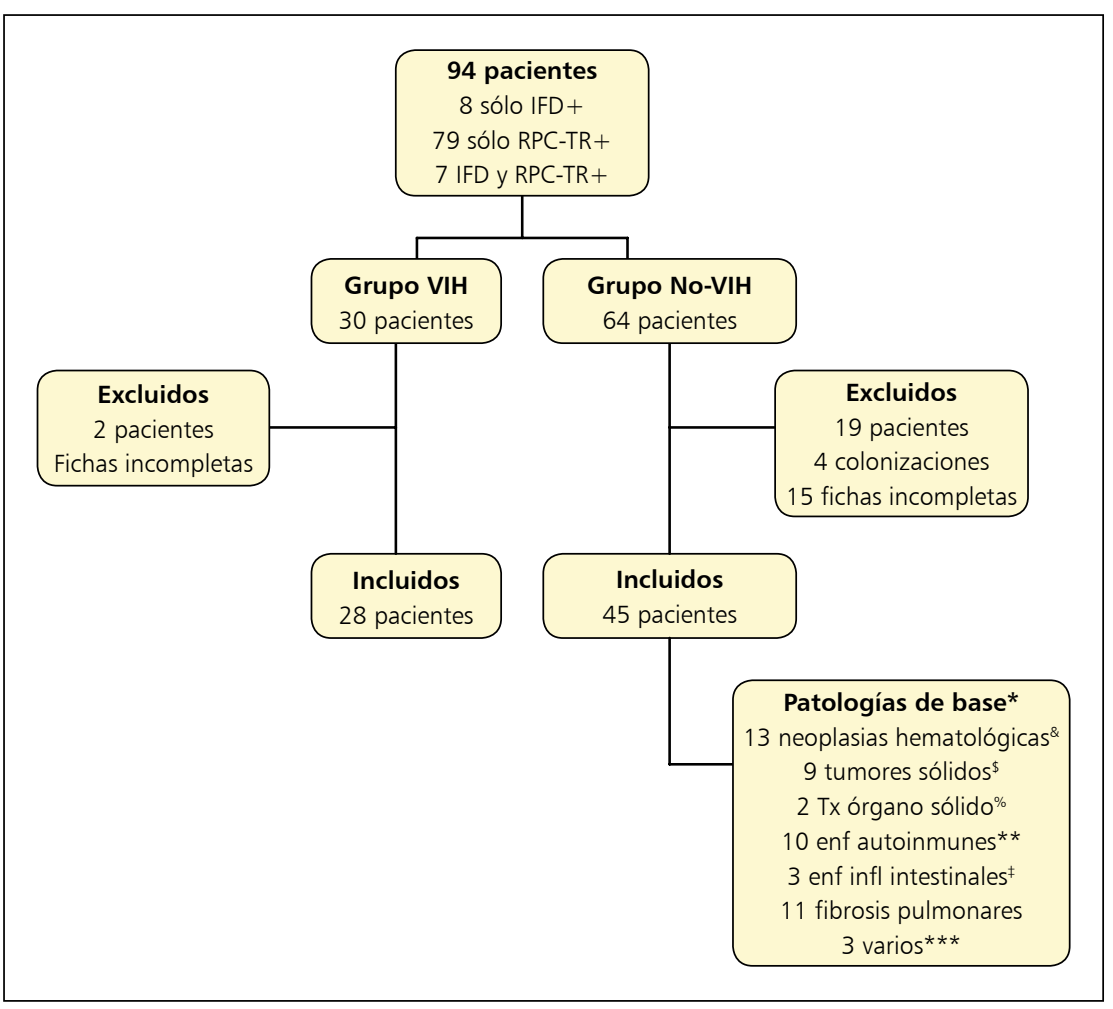

Figura 1. Algoritmo de selección de pacientes incluidos y clasificación de acuerdo a enfermedad de base. *Algunos pacientes presentaban más de una patología de base. ${ }^{2} 2$ Leucemias linfáticas crónicas, 4 leucemias linfáticas agudas, 4 linfomas no Hodgkin, 3 mielomas múltiples. \$2 cánceres de mama, 3 cánceres pulmonares, 1 cáncer endometrial, 1 cáncer gástrico, 2 sarcomas. \% 1 transplante renal, 1 transplante hepático. ${ }^{* *} 2$ artritis reumatoidea, 2 dermatomiositis, 1 polimiositis, 2 enfermedades indiferenciadas del tejido colectivo, 2 lupus eritematoso sistémico, 1 polimialgia reumática. ${ }^{\ddagger} 2$ enfermedades de Crohn, 1 colitis ulcerosa. ${ }^{* *} 1$ insuficiencia renal crónica en peritoneodiálisis, 1 daño hepático crónico, 1 miopatía por cuerpos de inclusión.

Tabla 1. Comparación de las características clínicas de pacientes, con y sin infección por $\mathrm{VIH}$, y neumonía por $P$. jiroveci

\begin{tabular}{|c|c|c|c|}
\hline Variable & $\begin{array}{c}\text { VIH } \\
\mathbf{n}=28(\%)\end{array}$ & $\begin{array}{c}\text { No VIH } \\
n=45(\%)\end{array}$ & Valor $p$ \\
\hline Fiebre & $27(96)$ & $42(93)$ & 1 \\
\hline Temperatura $\geq 39^{\circ} \mathrm{C}$ & $15(54)$ & $13(29)$ & 0,06 \\
\hline Tos & $26(93)$ & $33(73)$ & 0,07 \\
\hline Sin expectoración & $16(60)$ & $11(33)$ & 0,03 \\
\hline Expectoración mucosa & $9(35)$ & $10(30)$ & 0,94 \\
\hline Expectoración muco-purulenta & $1(4)$ & $12(36)$ & $<0,01$ \\
\hline Disnea & $22(79)$ & $41(91)$ & 0,16 \\
\hline En reposo & $8(36)$ & $31(76)$ & $<0,01$ \\
\hline De esfuerzo mínimo & $7(32)$ & $8(20)$ & 0,43 \\
\hline De esfuerzo máximo & $7(32)$ & $2(5)$ & $<0,01$ \\
\hline Taquipnea & $23(82)$ & $40(89)$ & 0,49 \\
\hline Examen pulmonar alterado & $21(75)$ & $43(96)$ & 0,02 \\
\hline
\end{tabular}


Tabla 2. Agentes microbiológicos causantes de infección pulmonar concomitante con neumonía por $P$. jiroveci en pacientes, con y sin infección por VIH

$\begin{array}{lccc}\text { Variable } & \begin{array}{c}\text { VIH } \\ \mathbf{n}=\mathbf{2 8}(\%)\end{array} & \begin{array}{c}\text { No VIH } \\ \mathbf{n = 4 5}(\%)\end{array} & \text { Valor } \mathbf{p} \\ \begin{array}{l}\text { Infección pulmonar concomitante por } \\ \text { otros agentes infecciosos* }\end{array} & 5(17,9) & 19(42,2) & 0,03 \\ \text { Virus* }^{*} & 2(7,1) & 9(20) & 0,18 \\ \text { Bacterias } & 2(7,1) & 6(13,3) & 0,70 \\ \text { Hongos }^{\&} & 1(3,6) & 6(13,3) & 0,23 \\ \text { Micobacterias }^{\$} & 2(7,1) & 0(0) & 0,14\end{array}$

Notas: "En algunos casos concomitancia con más de un microorganismo; * dengue, influenza, parainfluenza, $\mathrm{CMV}$; ${ }^{8}$ Aspergillus sp; ${ }^{\$} M$. tuberculosis, $M$. avium complex.

Tabla 3: Comparación de parámetros de laboratorio y radiográficos entre pacientes, con y sin infección por VIH, y neumonía por P. jiroveci

\begin{tabular}{|c|c|c|c|}
\hline Variable & $\begin{array}{c}\text { VIH } \\
n=28(\%)\end{array}$ & $\begin{array}{c}\text { No VIH } \\
n=45(\%)\end{array}$ & Valor $p$ \\
\hline \multicolumn{4}{|l|}{ Bioquímica } \\
\hline LDH U/L [mediana (rango)] & $410(150-792)$ & $415(113-2.050)$ & 0,21 \\
\hline PCR mg/dL [mediana (rango)] & $9(1,4-33)$ & $11,4(0,7-36)$ & 0,50 \\
\hline Recuento leucocitos $\left(\times 10^{3} / \mathrm{mm}^{3}\right)$ [mediana (rango)] & $7,0(2,8-16,1)$ & $8,5(0,1-129,0)$ & 0,25 \\
\hline Recuento linfocitos $\left(\times 10^{3} / \mathrm{mm}^{3}\right)$ [mediana (rango)] & $0,72(0,1-2,4)$ & $0,7(0-81,6)$ & 0,3 \\
\hline \multicolumn{4}{|l|}{ Diagnóstico radiológico } \\
\hline Radiografía de tórax patológica & $26(93)$ & $45(100)$ & 0,14 \\
\hline Infiltrados intersticiales difusos bilaterales & $23(88)$ & $16(36)^{\#}$ & $<0,01$ \\
\hline Consolidaciones & $2(8)$ & $28(62)^{\#}$ & $<0,01$ \\
\hline Nódulos pulmonares y/o efusión pleural & $1(4)$ & 2 (4) & 1 \\
\hline \multicolumn{4}{|l|}{ Diagnóstico microbiológico } \\
\hline \multicolumn{4}{|l|}{ Técnica diagnóstica } \\
\hline RPC-TR & $19(68)$ & 42 (93) & $<0,01$ \\
\hline IFD & $9(32)$ & $3 \quad(7)$ & $<0,01$ \\
\hline \multicolumn{4}{|l|}{ Tipo de muestra } \\
\hline Invasora (LBA) & $6(21)$ & $27 \quad(60)$ & $<0,01$ \\
\hline No invasora\& & $22(79)$ & $18 \quad(40)$ & $<0,01$ \\
\hline \multicolumn{4}{|c|}{$\begin{array}{l}\text { Notas: LDH: lactato deshidrogenasa; PCR: proteína C reactiva; RPC-TR: reacción de polimerasa en } \\
\text { cadena, en tiempo real; IFD: inmunoinfluorescencia directa; LBA: lavado bronquio-alveolar; \# Un } \\
\text { paciente con infiltrados y consolidaciones concomitantes; }{ }^{\circledR} \text { Expectoración, gárgara, aspirado naso- } \\
\text { faríngeo, aspirado endotraqueal. }\end{array}$} \\
\hline
\end{tabular}

logró exclusivamente por técnica de RPC-TR en 9 de ellos $(64,3 \%)$ mientras que en los pacientes del grupo no infectado por VIH con estudio por ambas técnicas, el diagnóstico fue posible sólo por técnica de RPC-TR en 32 de ellos $(94,1 \%), p=0,01$.

\section{Terapia específica}

En ambos grupos el tratamiento de elección fue cotrimoxazol con asociación o necesidad de cambio a clindamicina y/o primaquina en algunos casos, sin diferencias entre ambos grupos. La decisión del/(los) esquema(s) antimicrobiano(s) utilizado(s) fue elección del médico tratante. Salvo antecedente de alergia a sulfas, el tratamiento preferido para el inicio de la terapia antimicrobiana fue cotrimoxazol y, dependiendo del grado de respuesta clínica o desarrollo de alergia, se realizó adición o cambio de tratamiento a clindamicina \pm primaquina. Tampoco hubo diferencias en cuanto al uso de corticosteroides (hidrocortisona/prednisona) como parte del tratamiento de la infección por $P$. jiroveci entre ambos grupos $(67,9$ vs $80 \%, p=0,24)$. Los pacientes no infectados por VIH tuvieron mayor requerimiento de UCI ( 58 vs $25 \%, \mathrm{p}<0,01)$ y ventilación mecánica (56 vs $11 \%, \mathrm{p}<0,01)$, falleciendo $26(57,8 \%)$ pacientes con 15 muertes atribuibles $(33,3 \%)$ vs ningún fallecido en el grupo con infección por VIH ( $\mathrm{p}<0,01)$ (Tabla 4). Al buscar otras causas que pudieran influir en la mayor mortalidad del grupo no infectado por VIH, no se encontró correlación entre la presencia de co-infección por otros agentes infecciosos y mortalidad atribuible: ésta se observó en $31,6 \%$ de los pacientes co-infectados vs $34,6 \%$ de los pacientes sin co-infección, $\mathrm{p}=1$ ); mientras que la única patología de base que se correlacionó con un riesgo significativamente aumentado de mortalidad atribuible fue la fibrosis pulmonar, con $75 \%$ de mortalidad atribuible vs $24,3 \%$ de los pacientes sin fibrosis pulmonar $(p=0,01)$. En la Figura 2 se grafican las curvas de sobrevida al término del tratamiento antimicrobiano, tanto para todos los eventos (3a) como para las muertes atribuibles a la neumonía por $P$. jiroveci ( $3 \mathrm{~b}$ ) evidenciando la significativa mayor mortalidad de los pacientes no infectados por VIH.

\section{Discusión}

Nuestros resultados confirman descripciones de la literatura médica internacional respecto a las dificultades diagnósticas, gravedad y pronóstico entre las neumonías por $P$. jiroveci de pacientes con infección por VIH y las de pacientes no infectados ${ }^{13,16,17,20}$. En este último grupo destacan el mayor requerimiento de UCI, de VM y de mortalidad atribuible.

A la luz de nuestros resultados, se puede plantear que la peor evolución de los pacientes no infectados por VIH es probablemente de origen multifactorial participando condiciones propias del hospedero, co-infecciones presentes y dificultades diagnósticas que determinan retraso en el inicio oportuno de tratamiento.

Tal como en nuestros resultados, en la literatura 
científica se destaca la mayor edad y el mayor número de co-morbilidades de los pacientes no infectados por VIH lo que determinaría un mayor riesgo de evolución desfavorable ${ }^{13,16,17}$.

Entre los mecanismos fisiopatológicos subyacentes en el curso de la neumonía, se describen diferencias entre ambos grupos dadas por una mayor presencia de células inflamatorias a nivel alveolar, con menor carga infectante en el grupo no infectado por VIH, al contrario de lo que ocurre en los pacientes seropositivos. Esta inflamación pulmonar neutrofílica desencadenaría daño alveolar difuso, alteración del intercambio gaseoso y falla respiratoria. Limper y cols., describieron que la falla respiratoria y muerte estarían más estrechamente relacionadas con el grado de inflamación pulmonar que con la carga de microorganismos por lo que, paradójicamente, la mayor capacidad inflamatoria de los pacientes no infectados por VIH incidiría en el peor pronóstico ${ }^{25}$.

En nuestro estudio, los pacientes no infectados por VIH presentaron más frecuentemente co-infecciones pulmonares por otros microorganismos, encontrándose que un tercio de ellos se diagnosticó la enfermedad por $P$. jiroveci en forma posterior a otra infección pulmonar en curso. Aun cuando hay pocos estudios que hagan alusión a la concomitancia de otras infecciones pulmonares ${ }^{16,26,27}$ y su efecto en el pronóstico, es planteable que la doble infección generaría mayor inflamación e injuria pulmonar, incidiendo en una peor evolución; en nuestro estudio no logramos determinar una correlación entre co-infección y un riesgo aumentado de mortalidad.

La complejidad en obtener el diagnóstico en el grupo no infectado por $\mathrm{VIH}$, reflejado en la necesidad de técnicas invasoras y mayor demora en la confirmación etiológica, contribuye al retardo en el inicio de tratamiento y por ende, incide en el resultado final. Influirían en esta complejidad: la falta o baja sospecha clínica de esta infección en población no infectada por VIH, lo poco específico de los síntomas y signos, y el menor rendimiento de la IFD explicada por la menor carga infectante de los pacientes no infectados por $\mathrm{VIH}^{25}$. En una serie de 448 pacientes no infectados por VIH, con infiltrados pulmonares, la neumonía por $P$. jiroveci fue diagnosticada en $11,8 \%$ de éstos, de los cuales sólo 74\% fueron detectados por técnicas convencionales (IFD o tinción de Giemsa) vs 91\% de diagnóstico por RPC (28). Por otra parte, también es importante destacar que pese a que el LBA presenta mejor rendimiento diagnóstico, no siempre es factible realizarlo por la gravedad de la insuficiencia respiratoria. Una alternativa a considerar es la inducción de esputo con soluciones salinas hipertónicas, en las que se describen rendimientos diagnósticos de 50 a $90 \%{ }^{29}$ o las muestras de gárgara para estudio con RPC con sensibilidad y especificidad descritas mayores a $90 \%$, respectivamente ${ }^{23,24}$.
Tabla 4. Comparación de tratamiento y evolución clínica de pacientes, con y sin infección por VIH y neumonía por P. jiroveci

\begin{tabular}{|c|c|c|c|}
\hline Variable & $\begin{array}{c}\text { VIH } \\
n=28(\%)\end{array}$ & $\begin{array}{c}\text { No-VIH } \\
n=45(\%)\end{array}$ & Valor $p$ \\
\hline \multicolumn{4}{|l|}{ Tratamiento antimicrobiano } \\
\hline Cotrimoxazol & $21(75)$ & $33(73)$ & 0,90 \\
\hline Cotrimoxazol $+[$ clindamicina \pm primaquina $]$ & $5(18)$ & $11(24)$ & 0,71 \\
\hline Clindamicina \pm primaquina & $1(4)$ & $1(2)$ & 1 \\
\hline Pafuramidina maleato* & $1(4)$ & $0 \quad(0)$ & 0,38 \\
\hline Tratamiento corticosteroidal & $19(68)$ & $36(80)$ & 0,24 \\
\hline \multicolumn{4}{|l|}{ Evolución } \\
\hline Requerimiento de ingreso a UCI & $7(25)$ & $26(58)$ & $<0,01$ \\
\hline Duración estadía en UCI [mediana (rango)] & $4 d[1-50 d]$ & $18 \mathrm{~d}[1-36 \mathrm{~d}]$ & 0,73 \\
\hline Requerimiento de VM & $3(11)$ & $25(56)$ & $<0,01$ \\
\hline Días en VM [mediana (rango)] & $3 d[1-47 d]$ & $13 d[1-36]$ & $<0,01$ \\
\hline Duración de la hospitalización [mediana (rango)] & $10 d[4-113 d]$ & $18 d[3-60 d]$ & 0,28 \\
\hline \multicolumn{4}{|l|}{ Mortalidad } \\
\hline Mortalidad global & $0(0)$ & $26(58)$ & $<0,01$ \\
\hline Mortalidad atribuible & $0 \quad(0)$ & $15(33)$ & $<0,01$ \\
\hline
\end{tabular}

Notas: UCI: unidad de paciente crítico; VM: ventilación mecánica; * Uso en contexto de ensayo clínico randomizado controlado.

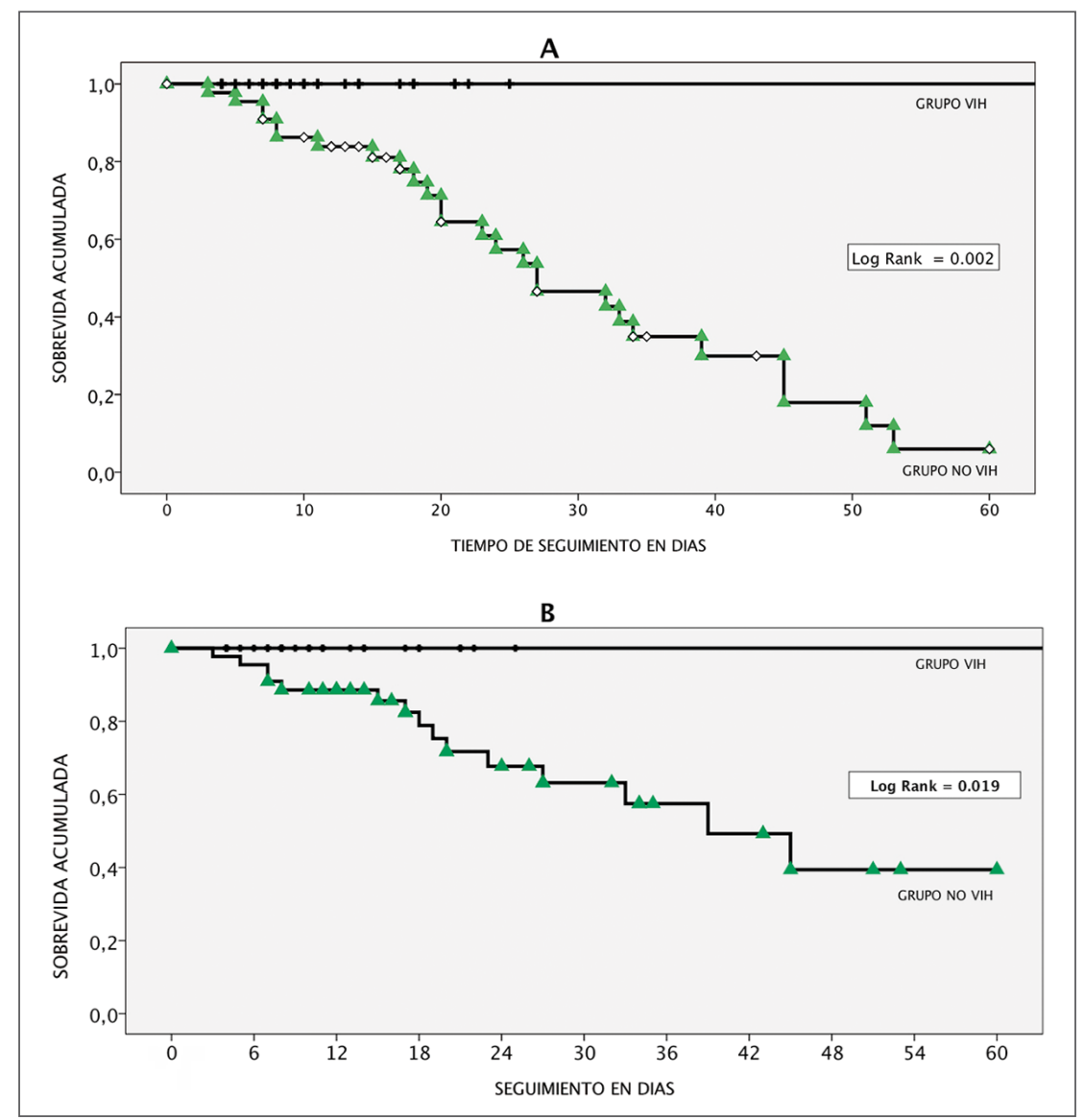

Figura 2. Curvas Kaplan-Meier de supervivencia acumulada a 60 días de seguimiento. A) Considerando el total de las muertes. B) Considerando sólo las muertes atribuibles a la neumonía por $P$. jirovecii. 
Uno de los potenciales riesgos de la implementación de técnicas de alta sensibilidad y especificidad como es la RPC, resulta ser el sobre diagnóstico de pneumocistosis en casos de colonización de la vía respiratoria. En la literatura científica se reportan incidencias de colonización de hasta $20 \%$, independiente de la situación inmunitaria $^{30,31}$ e incluso mayores a $60 \%$ en estudios anatomo-patológicos ${ }^{32}$. La verdadera implicancia de una colonización por $P$. jiroveci no está clara. Los individuos colonizados jugarían un rol importante como reservorios y potenciales trasmisores del patógeno a otros hospederos susceptibles, pero se cree que también experimentarían efectos a nivel personal: la sola colonización podría gatillar una respuesta pro-inflamatoria pulmonar similar a la de una neumonía por $P$. jiroveci activa ${ }^{33}$. Por lo anterior, y pese a las dudas que pudiera genera la interpretación de colonización vs infección, parece razonable utilizar técnicas diagnósticas de alta eficiencia para evitar así el retraso del inicio de tratamiento específico en caso de ser necesario.

Por todo lo anteriormente señalado, nos parece muy importante la optimización de las estrategias de prevención de la neumonía por $P$. jiroveci. Mientras para pacientes con infección por VIH existen guías claras que recomiendan el uso de profilaxis en pacientes con recuentos de LTCD4 < 200 cél $/ \mathrm{mm}^{3}{ }^{34}$, la información es menos homogénea respecto a la profilaxis en pacientes inmunocomprometidos no infectados por VIH. Hay publicadas guías de profilaxis de neumonía por $P$. jiroveci en pacientes con cáncer, trasplantes de precursores hematopoyéticos y trasplantes de órganos sólidos ${ }^{35,36}$; pero no las hay para pacientes con enfermedades reumatológicas recibiendo terapia inmunosupresora, aunque debiera considerarse un grupo de alto riesgo, dado que está descrito que están expuestos al desarrollo de pneumocistosis aquellos que reciben dosis equivalentes a más de $20 \mathrm{mg}$ diarios de prednisona por más de dos meses ${ }^{37}$. De acuerdo al meta-análisis de Green y cols., la profilaxis para neumonía por $P$. jiroveci es recomendable cuando el riesgo es superior a $3,5 \%$, pues el beneficio es mayor que los efectos adversos ${ }^{36}$. Cabe destacar la alta mortalidad encontrada en pacientes con fibrosis pulmonar, la que probablemente se explica porque la inflamación pulmonar gatillada por $P$. jiroveci $i$ afecta seriamente la ya mermada reserva funcional de estos pacientes y por ende, creemos que es otra población en la que se debiera plantear el uso de profilaxis.

Nuestro estudio presenta algunas limitaciones: diseño retrospectivo; por el eventual sesgo de inclusión ya que la investigación etiológica de los casos de neumonía por $P$. jiroveci detectados fue realizada en base a la solicitud de los tratantes y no a un protocolo estandarizado, lo que podría haber llevado al reconocimiento de los casos más graves excluyendo casos leves y de esta manera pudiera haber incidido en la peor evolución del grupo no infectados por VIH. De todas formas creemos que estas limitaciones no afectan la validez de los resultados obtenidos dado que éstos se correlacionan de buena forma con los reportados en la literatura médica internacional.

En conclusión, nuestro estudio muestra que la pneumocistosis en pacientes no infectados por VIH es de mayor gravedad, ofrece más dificultades diagnósticas y presenta mayor mortalidad que en pacientes con infección por VIH, por lo que en el primer grupo debería plantearse la optimización de estrategias de prevención y diagnóstico precoz mediante un alto nivel de sospecha, incluyendo el uso de RPC-TR, además de investigar estrategias de intervenciones terapéuticas que permitan mejorar el pronóstico actual.

\section{Resumen}

Introducción: Pneumocystis jiroveci puede causar neumonía en pacientes inmunocomprometidos de cualquier etiología, pero las diferencias clínicas y pronósticas entre inmunocomprometidos por VIH y por otras causas han sido poco exploradas. Objetivo: Comparar las características clínicas, de laboratorio y pronóstico de neumonía por $P$. jiroveci en pacientes inmunocomprometidos por infección VIH versus no infectados por VIH. Métodos: Análisis retrospectivo de casos confirmados de neumonía por $P$. jiroveci en adultos con infección por VIH y no infectados, entre los años 2005 y 2007. Resultados: Se incluyeron 28 pacientes infectados por VIH y 45 no infectados, con neumonía por $P$. jiroveci confirmada. La población no infectada por VIH presentaba mayor edad (65 vs 36,2 años, $\mathrm{p}<0,01$ ), menor duración de síntomas previos a la consulta (7 [121] vs 14 [2-45] días, $p<0,01)$, mayor requerimiento de técnica invasora $(60$ vs $21 \%, \mathrm{p}<0,01)$ y estudio molecular (93 vs $68 \%, \mathrm{p}<0,01$ ) para confirmación diagnóstica, mayor requerimiento de camas críticas (58 vs $25 \%, \mathrm{p}<0,01$ ), y ventilación mecánica (56 vs $11 \%$, $\mathrm{p}<0,01$ ), con mayor mortalidad atribuible (33 vs $0 \%$, $\mathrm{p}<0,01)$. Conclusiones: La neumonía por P. jiroveci en pacientes inmunocomprometidos no infectados por VIH ofrece más dificultades diagnósticas y presenta mayor gravedad y mortalidad que en pacientes con infección por VIH; por esto, es mandatario optimizar los procesos diagnóstico y terapéutico en esta población. 


\section{Referencia bibliográfícas}

1.- Thomas C F Jr, Limper A H. Pneumocystis pneumonia. N Engl J Med 2004; 350 (24): 2487-98.

2.- Sepkowitz K A. Opportunistic infections in patients with and patients without acquired immunodeficiency syndrome. Clin Infect Dis 2002; 34(8): 1098-107.

3.- Radisic M, Lattes R, Chapman J F, del Carmen Rial M, Guardia O, Seu F, et al. Risk factors for Pneumocystis carinii pneumonia in kidney transplant recipients: a case-control study. Transpl Infect Dis 2003; 5 (2): 84-93.

4.- Gerrard J G. Pneumocystis carinii pneumonia in HIV-negative immunocompromised adults. Med J Aust 1995; 162 (5): 233-5.

5.- Yoo J H, Lee D G, Choi S M, Choi J H, Park Y H, Kim Y J, et al. Infectious complications and outcomes after allogeneic hematopoietic stem cell transplantation in Korea. Bone Marrow Transplant 2004; 34 (6): 497-504.

6.- De Castro N, Neuville S, Sarfati C, Ribaud P, Derouin F, Gluckman E, et al. Occurrence of Pneumocystis jiroveci pneumonia after allogeneic stem cell transplantation: a 6-year retrospective study. Bone Marrow Transplant. 2005; 36 (10): 879-83.

7.- Torres H A, Chemaly R F, Storey R, Aguilera E A, Nogueras G M, Safdar A, et al. Influence of type of cancer and hematopoietic stem cell transplantation on clinical presentation of Pneumocystis jiroveci pneumonia in cancer patients. Eur J Clin Microbiol Infect Dis 2006; 25(6): 382-8.

8.- Sepkowitz K A, Brown A E, Telzak E E, Gottlieb S, Armstrong D. Pneumocystis carinii pneumonia among patients without AIDS at a cancer hospital. JAMA 1992; 267 (6): 832-7.

9.- Hughes W T, Feldman S, Aur R J, Verzosa M S, Hustu H O, Simone J V. Intensity of immunosuppressive therapy and the incidence of Pneumocystis carinii pneumonitis. Cancer. 1975; 36 (6): 2004-9.

10.- Kulke M H, Vance E A. Pneumocystis carinii pneumonia in patients receiving chemotherapy for breast cancer. Clin Infect Dis 1997; 25 (2): 215-8.

11.- Johnson H D, Johnson W W. Pneumocystic carinii pneumonia in children with cancer. Diagnosis and treatment. JAMA 1970; 214 (6): 1067-73.

12.- Walzer P D, Perl D P, Krogstad D J, Rawson P G, Schultz M G. Pneumocystis carinii pneumonia in the United States. Epidemiologic, diagnostic, and clinical features. Ann Intern Med 1974; 80 (1): 83-93.

13.- Mansharamani N G, Garland R, Delaney D,
Koziel H. Management and outcome patterns for adult Pneumocystis carinii pneumonia, 1985 to 1995: comparison of HIV-associated cases to other immunocompromised states. Chest 2000; 118 (3): 704-11.

14.- Roblot F, Godet C, Le Moal G, Garo B, Faouzi Souala M, Dary M, et al. Analysis of underlying diseases and prognosis factors associated with Pneumocystis carinii pneumonia in immunocompromised HIV-negative patients. Eur J Clin Microbiol Infect Dis 2002; 21(7): 523-31.

15.- Arend S M, Kroon F P, van't Wout J W. Pneumocystis carinii pneumonia in patients without AIDS, 1980 through 1993. An analysis of 78 cases. Arch Intern Med 1995; 155 (22): 2436-41.

16.- Ewig S, Bauer T, Schneider C, Pickenhain A, Pizzulli L, Loos U, et al. Clinical characteristics and outcome of Pneumocystis carinii pneumonia in HIV-infected and otherwise immunosuppressed patients. Eur Respir J 1995; 8 (9): 1548-53.

17.- Nuesch R, Bellini C, Zimmerli W. Pneumocystis carinii pneumonia in human immunodeficiency virus (HIV)-positive and HIV-negative immunocompromised patients. Clin Infect Dis 1999; 29 (6): 1519-23.

18.- Kovacs J A, Hiemenz J W, Macher A M, Stover D, Murray H W, Shelhamer J, et al. Pneumocystis carinii pneumonia: a comparison between patients with the acquired immunodeficiency syndrome and patients with other immunodeficiencies. Ann Intern Med 1984; 100 (5): 663-71.

19.- Edman J C, Edman U, Cao M, Lundgren B, Kovacs J A, Santi D V. Isolation and expression of the Pneumocystis carinii dihydrofolate reductase gene. Proc Natl Acad Sci USA; 86 (22): 8625-9.

20.- Li M C, Lee N Y, Lee C C, Lee H C, Chang C M, Ko WC. Pneumocystis jiroveci pneumonia in immunocompromised patients: Delayed diagnosis and poor outcomes in nonHIV-infected individuals. J Microbiol Immunol Infect 2012 Oct 10.

21.- Pérez C, Almendarez C, Oddo D, Sirhan M, Labarca J, Acuna G, et al. Pneumonia caused by Pneumocystis carinii in immunodepressed patients: clinical picture, treatment, and prognosis. Rev Med Chile 1994; 122 (2): 154-8.

22.- Pérez C, Müller C, Castillo Y, Mella S, Acuña G, Labarca J. Neumonía por Pneumocystis carinii: factores predisponentes, manifestaciones clínicas y respuesta al tratamiento. Rev Chilena Infectol 1999; 16: 296-301.

23.- Fischer S, Gill VJ, Kovacs J, Miele P, Keary J, Silcott V, et al. The use of oral washes to diagnose Pneumocystis carinii pneumonia: a blinded prospective study using a polymerase chain reaction-based detection system. J Infect Dis 2001; 184 (11): 1485-8.

24.- Larsen H H, Huang L, Kovacs J A, Crothers K, Silcott V A, Morris A, et al. A prospective, blinded study of quantitative touch-down polymerase chain reaction using oral-wash samples for diagnosis of Pneumocystis pneumonia in HIV-infected patients. J Infect Dis 2004; 189 (9): 1679-83.

25.- Limper A H, Offord K P, Smith T F, Martin W J, 2nd. Pneumocystis carinii pneumonia. Differences in lung parasite number and inflammation in patients with and without AIDS. Am Rev Respir Dis 1989; 140 (5): 1204-9.

26.- Kaira K, Shinomiya Y, Takahashi Y, Iida T, Yanagitani N, Sunaga N, et al. Coinfection of invasive pulmonary aspergillosis and Pneumocystis jiroveci pneumonia in a non-HIV patient. Acta Med Okayama 2007; 61 (4): 235-8.

27.- Matsumura Y, Shindo Y, Iinuma Y, Yamamoto M, Shirano M, Matsushima A, et al. Clinical characteristics of Pneumocystis pneumonia in non-HIV patients and prognostic factors including microbiological genotypes. BMC Infect Dis 2011; 11: 76.

28.- Azoulay E, Bergeron A, Chevret S, Bele N, Schlemmer B, Menotti J. Polymerase chain reaction for diagnosing Pneumocystis pneumonia in non-HIV immunocompromised patients with pulmonary infiltrates. Chest 2009; 135 (3): 655-61.

29.- Shelhamer J H, Gill V J, Quinn T C, Crawford S W, Kovacs J A, Masur H, et al. The laboratory evaluation of opportunistic pulmonary infections. Ann Intern Med 1996; 124 (6): 585-99.

30.- Sing A, Trebesius K, Roggenkamp A, Russmann H, Tybus K, Pfaff F, et al. Evaluation of diagnostic value and epidemiological implications of PCR for Pneumocystis carinii in different immunosuppressed and immunocompetent patient groups. J Clin Microbiol 2000; 38 (4): 1461-7.

31.- Medrano F J, Montes-Cano M, Conde M, de la Horra C, Respaldiza N, Gasch A, et al. Pneumocystis jirovecii in general population. Emerg Infect Dis. 2005 Feb; 11(2): 245-50.

32.- Ponce C A, Gallo M, Bustamante R, Vargas S L. Pneumocystis colonization is highly prevalent in the autopsied lungs of the general population. Clin Infect Dis 2010; 50 (3): 347-53.

33.- Morris A, Wei K, Afshar K, Huang L. Epidemiology and clinical significance of Pneumocystis colonization. J Infect Dis 2008; 197 (1): 10-7. 
34.- Kaplan J E, Benson C, Holmes K H, Brooks J T, Pau A, Masur H. Guidelines for prevention and treatment of opportunistic infections in HIV-infected adults and adolescents: recommendations from CDC, the National Institutes of Health, and the HIV Medicine Association of the Infectious Diseases Society of America. MMWR Recomm Rep 2009; 58 (RR-4): 1-207; quiz CE1-4.
35.- Guidelines for preventing opportunistic infections among hematopoietic stem cell transplant recipients. Recommendations of CDC, the Infectious Disease Society of America, and the American Society of Blood and Marrow Transplantation. Cytotherapy 2001; 3 (1): 41-54.

36.- Green H, Paul M, Vidal L, Leibovici L. Prophylaxis of Pneumocystis pneumonia in immunocompromised non-HIV-infected patients: systematic review and meta-analysis of randomized controlled trials. Mayo Clin Proc 2007; 82 (9): 1052-9.

37.- Yale S H, Limper A H. Pneumocystis carinii pneumonia in patients without acquired immunodeficiency syndrome: associated illness and prior corticosteroid therapy. Mayo Clin Proc 1996; 71 (1): 5-13. 